\title{
On the effective interactions between like-charged macromolecules
}

\author{
Emmanuel Trizac * \\ Laboratoire de Physique Théorique (Unité Mixte de Recherche UMR 8627 du CNRS), \\ Bâtiment 210, Université de Paris-Sud, 91405 Orsay Cedex, France
}

(November 20, 2018)

\begin{abstract}
We investigate, within a local density functional theory formalism, the interactions between likecharged polyions immersed in a confined electrolyte. We obtain a simple condition for a repulsive effective pair potential, that can be related to the thermodynamic stability criterion of the uncharged counterpart of microscopic species constituting the electrolyte. Under the same condition, the phenomenon of charge inversion (over-charging), where the polyion bare charge is over-screened by its electric double layer, is shown to be impossible. These results hold beyond standard mean-field theories (such as Poisson-Boltzmann or Modified Poisson-Boltzmann approaches).
\end{abstract}

PACS numbers: 05.70.Np, 64.10+h, 82.60Lf, 82.70.Dd

Electrostatic forces play a key role in determining the stability, phase and structural properties of colloids or macroions suspended in polar solvents (usually aqueous media). Such suspensions are ubiquitous in many biological and technologically important systems, from superabsorbants or water-soluble paints to DNA solutions. Their behavior is however far from being well understood, despite an intense theoretical effort over the last fifty years.

Upon dispersion in water, mesoscopic colloidal polyions bearing ionizable groups gain a (large) bare charge, and strongly attract counterions while repelling coions, thus giving rise to electric double layers characterized by strong inhomogeneities in the local density of these microions in the vicinity of the polyions. From the electrostatic part of the traditional Derjaguin-Landau-Verwey-Overbeek (DLVO) theory [1], the effective interactions (solvent and microion mediated) between two like-charged colloidal particles are expected to be repulsive [2], whereas experiments [3] and numerical simulations 伍 provide evidence for attraction, particularly within confined geometries (e.g. when the two macroions are close to a charged wall or between two glass plates).

We consider a mixture of $N$ microions, where species $\alpha$ has charge number $z_{\alpha}$ and local density $c_{\alpha}(\mathbf{r})$. Disregarding the molecular nature of the solvent, considered to be a confined electrolyte of dielectric permittivity $\varepsilon$, the effective interactions between two like-charge colloids are then analyzed within a generic local density functional theory. We show that the convexity of the free energy density with respect to the microions densities is a sufficient condition for having repulsive effective interactions and for preventing charge inversion. The physical implications of this result are considered in the final discussion (in particular the repulsion "often" follows from the thermodynamic stability criterion of the corresponding uncharged mixture of micro-species). The proof makes use of the results established in Refs. [5 7], and considers a geometry which encompasses many cases of experimental relevance: the confining region $\mathcal{R}$ is a cylinder of arbitrary cross section and length $2 L$ (in the limit of large $L$ ) while the medium $\mathcal{R}^{\prime}$ outside $\mathcal{R}$ is a dielectric continuum of permittivity $\varepsilon^{\prime}$ with the possibility of a uniform density of surface charges $\sigma$ on the boundary $\partial \mathcal{R}$. The standard Neuman and Dirichlet boundary conditions (constant normal electric field and constant potential respectively) belong to the above class, and the dielectric medium in $\mathcal{R}^{\prime}$ may contain an electrolyte solution (this possibility was not considered in Refs [5.6]). We assume global electroneutrality in both $\mathcal{R}$ and $\mathcal{R}^{\prime}$ and the two macroions we shall focus on may be of arbitrary shape provided the electrostatic potential possesses mirror symmetry with respect to a plane $O x y$ between the macroions. Our approach holds irrespective of the specific boundary conditions to be applied on the macroions (constant charge or constant potential) and is independent of the sign of the surface charge $\sigma$.

Omitting, for the sake of simplicity, their temperature dependence, the local [8] density free energy functionals [9] considered here are of the form

$$
\mathcal{F}\left(\left\{c_{\alpha}\right\}\right)=\int_{\mathcal{R}} f\left(\left\{c_{\alpha}(\mathbf{r})\right\}\right) d \mathbf{r}+\frac{1}{2} \int_{\mathcal{R}} \rho_{c}(\mathbf{r}) G\left(\mathbf{r}, \mathbf{r}^{\prime}\right) \rho_{c}\left(\mathbf{r}^{\prime}\right) d \mathbf{r} d \mathbf{r}^{\prime},
$$

where the Helmholtz free energy density $f$ is a function of all $N$ microions densities and the local charge density includes the contributions from the macroions $q_{\mathcal{M}}(\mathbf{r})$ as well as from the microions:

$$
\rho_{c}(\mathbf{r})=\sum_{\alpha=1}^{N} z_{\alpha} e c_{\alpha}(\mathbf{r})+q_{\mathcal{M}}(\mathbf{r}) .
$$

*Electronic Address: Emmanuel.Trizac@th.u-psud.fr 
In Eq. (11), $G\left(\mathbf{r}, \mathbf{r}^{\prime}\right)$ denotes the Green's function such that the electrostatic potential $\psi(\mathbf{r})$, which is a solution of Poisson's equation $\nabla^{2} \psi=-(4 \pi / \varepsilon) \rho_{c}(\mathbf{r})$ with the required boundary conditions on the surface $\partial \mathcal{R}$, can be cast in the form

$$
\psi(\mathbf{r})=\int_{\mathcal{R}} \rho_{c}\left(\mathbf{r}^{\prime}\right) G\left(\mathbf{r}, \mathbf{r}^{\prime}\right) d \mathbf{r}^{\prime}
$$

$G$ reduces to the usual Coulomb potential in the absence of confinement. The density functional formulation provided by Eq. (11) encompasses a large class of modelizations, including a) the standard Poisson Boltzmann theory when the ideal gas expression $f=\sum_{\alpha} k T c_{\alpha}\left[\ln \left(c_{\alpha} \Lambda_{\alpha}^{3}\right)-1\right]$ is chosen for the free energy density, $\left.b\right)$ modified Poisson Boltzmann theories derived to account for steric effects [10] or more general non-electrostatic interactions [11], and $c$ ) recent improvements over standard mean-field approximations incorporating ion-ion correlations in the form of a One Component Plasma correction [12]. This last case involves a free energy density which does not only depend on the local densities of microions but also on the elementary charge $e$, and will be examined in the concluding discussion. In general, the present analysis allows to incorporate correlation or non mean-field contributions, provided these effects translate into a local free energy density.

Minimization of the functional (1) subject to the normalization constraints $\int_{\mathcal{R}} c_{\alpha}(\mathbf{r}) d \mathbf{r}=N_{\alpha}$ yields the implicit relation between the densities $c_{\alpha}(\mathbf{r})$ and the electrostatic potential:

$$
\frac{\partial f}{\partial c_{\alpha}}+e z_{\alpha} \psi(\mathbf{r})=\mu_{\alpha}^{*}, \quad 1 \leq \alpha \leq N
$$

where $\mu_{\alpha}^{*}$ is the (electro)chemical potential of species $\alpha$. The effective force acting on a colloid is obtained from integration of the generalized Maxwell stress tensor $\boldsymbol{\Pi}$ over the surface $S$ of the macroion:

$$
\mathbf{F}=\oint_{S} \mathbf{\Pi} \cdot \mathbf{n} d S
$$

where the unit vector $\mathbf{n}$ points outward from the surface of integration. The local stress tensor follows from the computation of the reversible work required to locally deform the shape of the confining cylinder, with the result

$$
\boldsymbol{\Pi}=-\left[P+\frac{\varepsilon}{2} \nabla \psi \cdot \nabla \psi\right] \boldsymbol{I}+\varepsilon \boldsymbol{\nabla} \psi \otimes \boldsymbol{\nabla} \psi,
$$

where the osmotic pressure $P\left(\left\{c_{\alpha}\right\}\right)$, which depends on position via the local densities $c_{\alpha}(\mathbf{r})$, is the pressure that would be found in the medium in the absence of an electric field, with the same densities $c_{\alpha}(\mathbf{r})$ [13], and can be written as the Legendre transform of the free energy density:

$$
P\left(\left\{c_{\alpha}\right\}\right)=-f\left(\left\{c_{\alpha}\right\}\right)+\sum_{\alpha=1}^{N} c_{\alpha} \frac{\partial f}{\partial c_{\alpha}} .
$$

For any stress tensor of the form (6), it has been shown in Refs. [5] that the effective force $\mathbf{F}$ given by Eq. (5) has a component $F_{z}$ along the axis of the cylinder that can be cast in the form

$$
\begin{aligned}
F_{z} & =\int_{O x y}\left[P(\psi)_{z=0}-P(\psi)_{z=L}-\left(\psi_{z=0}-\psi_{z=L}\right) \frac{\partial P}{\partial \psi}\left(\psi_{z=L}\right)\right] d x d y \\
& +\frac{\epsilon}{2} \int_{O x y}\left(\nabla \psi_{z=0}-\nabla \psi_{z=L}\right)^{2} d x d y,
\end{aligned}
$$

where $\epsilon$ denotes $\varepsilon$ (respectively $\varepsilon^{\prime}$ ) for the part of the symmetry plane, denoted Oxy, belonging to $\mathcal{R}$ (respectively $\mathcal{R}^{\prime}$ ). In Equation (8), it is understood that the pressure depends on the potential via Eqs. (7) and (4), the latter giving sense to the notation $\partial P / \partial \psi$. The subsequent analysis is devoted to the proof that $P$ is a convex-up function of $\psi$ provided that the free energy density is itself convex-up with respect to the densities $\left\{c_{\alpha}\right\}$. Remembering the orientation convention chosen in Refs. [5], this in turn establishes the repulsive nature of the effective interactions $\left(F_{z}>0\right)$. From the direct differentiation of Eq. (7) we get

$$
\frac{\partial P}{\partial \psi}=-\rho_{c}(\mathbf{r})
$$


where use was made of the stationary condition (4). The relation (9) can be recovered from the mechanical equilibrium condition of a fluid element of microions: the balance between the electric force and the osmotic constraint acting on such a fluid element located at point $\mathbf{r}$ can indeed be written

$$
\begin{gathered}
-\nabla P=\rho_{c}(\mathbf{r}) \nabla \psi \\
\Leftrightarrow\left[\frac{\partial P}{\partial \psi}+\rho_{c}\right] \nabla \psi=\mathbf{0} .
\end{gathered}
$$

Equation (9) equivalently implies that the tensor $\boldsymbol{\Pi}$ is divergence free, as already invoked in Ref. [7] to obtain the osmotic equation of state in the specific case of a Modified Poisson Boltzmann theory.

The second derivative of the osmotic pressure,

$$
\frac{\partial^{2} P}{\partial \psi^{2}}=-e \sum_{\alpha} z_{\alpha} \frac{\partial c_{\alpha}}{\partial \psi}
$$

involves the quantities $\partial c_{\alpha} / \partial \psi$ which may be determined from the condition (4):

$$
\sum_{\beta=1}^{N} \frac{\partial^{2} f}{\partial c_{\alpha} \partial c_{\beta}} \frac{\partial c_{\beta}}{\partial \psi}=-e z_{\alpha}
$$

Assuming the free energy density to be a convex-up function of the $\left\{c_{\alpha}\right\}$ implies that the matrix $\boldsymbol{H}$ with elements

$$
H_{\alpha \beta} \equiv \frac{\partial^{2} f}{\partial c_{\alpha} \partial c_{\beta}}
$$

is positive definite. Its inverse $\boldsymbol{H}^{-1}$ is consequently positive definite from which we can invert relation (13):

$$
\frac{\partial c_{\alpha}}{\partial \psi}=-e \sum_{\beta=1}^{N}\left(\boldsymbol{H}^{-1}\right)_{\alpha \beta} z_{\beta}
$$

Upon substitution of Eq. (15) into (12) we finally obtain

$$
\frac{\partial^{2} P}{\partial \psi^{2}}=e^{2} \sum_{\alpha, \beta}\left(\boldsymbol{H}^{-1}\right)_{\alpha \beta} z_{\alpha} z_{\beta}>0
$$

The pressure $P$ is therefore a convex-up function of the electrostatic potential, which establishes the aforementioned connection between the convexity of $f$ and the sign of the polyion-polyion effective interactions. Note that the non convexity of the free energy density is a necessary though insufficient condition for the existence of attraction within a local density approximation formalism.

We now address the question of charge reversal, where the nominal charge of a bare polyion can be over-screened by its condensed counterions. This interesting phenomenon (occurring for instance in DNA salt systems [14]) requires, from Gauss's theorem, the vanishing of the normal component of the electric field $(-\mathbf{n} \cdot \boldsymbol{\nabla} \psi)$ on a closed surface with normal $\mathbf{n}$ around the polyion. Taking the gradient of Eq. (4) then implies, without any symmetry assumption

$$
\sum_{\beta=1}^{N} \frac{\partial^{2} f}{\partial c_{\alpha} \partial c_{\beta}}\left[\mathbf{n} \cdot \nabla \mathbf{c}_{\beta}\right]=0
$$

hence the existence of a vanishing eigenvalue [with eigenvector $\left(\mathbf{n} \cdot \boldsymbol{\nabla} c_{\beta}\right)_{\beta=1 \ldots N}$ ] of the stability matrix $\boldsymbol{H}$, which is impossible under the assumption of positive definiteness.

Within the framework of Poisson Boltzmann theory, the repulsive nature of the effective electrostatic force between two similar colloidal bodies [5] and the impossibility of charge reversal immediately follows from the previous analysis. However, if for the sake of analytical tractability and in the spirit of Ref. [15], the ideal free energy density is further Taylor expanded up to order $\mathcal{N}$ around the mean densities $\left\{\bar{c}_{\alpha}\right\}$, we obtain

$$
H_{\alpha \beta}=\frac{\delta_{\alpha \beta}}{\bar{c}_{\alpha}} \sum_{i=0}^{\mathcal{N}-2}\left(1-\frac{c_{\alpha}}{\bar{c}_{\alpha}}\right)^{i}=\left[1-\left(1-\frac{c_{\alpha}}{\bar{c}_{\alpha}}\right)^{\mathcal{N}-1}\right] \frac{\delta_{\alpha \beta}}{c_{\alpha}} .
$$


The truncated Hessian $\boldsymbol{H}$ is therefore positive definite and gives a repulsive force for even values of $\mathcal{N}$ (as in the non-linear Poisson-Boltzmann theory), while it can be non-positive definite and the force attractive for odd orders $\mathcal{N}$ provided the local density of one counterion species exceeds twice its mean density. This explains the repulsion within linearized Poisson-Boltzmann theory [16] (where linearization amounts to taking $\mathcal{N}=2$ [9]), and the attraction seen in Ref. [15] with an expansion pushed one order further and truncated after third order to account for the triplet interactions between two polyions and a charged wall.

We have shown that effective attractive pair potentials and over-charging are both ruled out under the assumption of positive definiteness for the stability matrix $\boldsymbol{H}$. This is a necessary requirement when the free energy density does not depend on the elementary charge $e$. The key ingredient here is the existence of a thermodynamically stable neutral mixture of micro-species described by the same density $f\left\{c_{\alpha}\right\}$ as its charged counterpart. On the other hand, the inclusion of correlations and/or fluctuations corrections is believed to be essential in order to describe the appearance of attractive interactions. If their correction to the mean-field electrostatic term $\int \rho_{c} G \rho_{c}$ in the right hand side of Eq. (11) is taken into account, and translated into an $e$-dependent free energy density [12,18], the thermodynamic stability condition is now the positive definiteness of the integral operator whose kernel is defined by

$$
\frac{\partial^{2} f}{\partial c_{\alpha} \partial c_{\beta}} \delta\left(\mathbf{r}-\mathbf{r}^{\prime}\right)+e^{2} z_{\alpha} z_{\beta} G\left(\mathbf{r}, \mathbf{r}^{\prime}\right)
$$

and it is in general impossible to find a locally neutral mixture described by the same density $f$. $\boldsymbol{H}$ need not therefore be positive definite. However, non convex-up densities $f$ such as the correction put forward by Stevens and Robbins 17. by extrapolation of the Monte Carlo data for the homogeneous One Component Plasma lead to an instability at high densities (the so called structuring catastrophe), and cannot be used in a thermodynamically stable way within a local density functional theory [12,18]. The recent attempt of Barbosa et al. [12] to circumvent this instability gives rise to a convex-up $f$, with results reproducing satisfactorily the ionic correlations present in Molecular Dynamics simulations. The present work indicates that the effective interactions are then necessarily repulsive and charge inversion impossible, regardless of the analytical complexity of the formalism.

To conclude, repulsive effective pair potentials seem generic within local density approximations. The description of attraction and over-charging thus requires involved density functional theories, and it is not sufficient to incorporate correlation effects in a local formulation. Our analysis points to the importance of non-local effects, that can be accounted for by weighted density functionals [17,18], or simpler approaches relying on non-electrostatic depletion interactions [19].

The author would like to thank J.L. Raimbault, D. Levesque, J.P. Hansen, B. Jancovici, H. Hilhorst, M. Deserno and D. Rowan for helpful discussions.

[1] B.V. Derjaguin and L. Landau, Acta Physicochim. URSS 14, 633 (1941); E.J.W. Verwey and J.Th.G. Overbeek, Theory of the Stability of Lyophobic Colloids (Elsevier, New York, 1948).

[2] J. Chakrabarti and H. Löwen, Phys. Rev. E 58, 3400 (1998); A. Denton and H. Löwen, Thin Solid Films 330, 7 (1998); K. Vondermasson, J. Bongers, A. Mueller and H. Versmold, Langmuir 10, 1351 (1994); J.C. Crocker and D.G. Grier, Phys. Rev. Lett. 73, 352 (1994).

[3] G.M. Kepler and S. Fraden, Phys. Rev. Lett. 73, 356 (1994); M.D. Carbajal-Tinoco, F. Castro-Román and J.L. ArauzLara, Phys. Rev. E 53, 3745 (1996); A.M. Larsen and D.G. Grier, Nature 385, 230 (1997); J.C. Crocker and D.G. Grier, Phys. Rev. Lett. 77, 1897 (1996); D.G. Grier, Nature 393, 621 (1998).

[4] N. Grønbech-Jensen, K.M. Beardmore and P. Pincus, Physica A 261, 74 (1998); E. Allahyarov, I. D'Amico and H. Löwen, Phys. Rev. E 60, 3199 (1999); P. Linse and V. Lobaskin, Phys. Rev. Lett. 83, 4208 (1999).

[5] J.C. Neu, Phys. Rev. Lett. 82, 1072 (1999).

[6] J.E. Sader and D.Y. Chan, J. Colloid Interface Sci. 213, 268 (1999). J.E. Sader and D.Y. Chan, Langmuir 16, 324 (2000).

[7] E. Trizac and J.L. Raimbault, Phys. Rev. E 60, 6530 (1999).

[8] The "locality" does not refer to the van der Waals like mean-field term $\int \rho_{c} G \rho_{c}$ but to the term $\int f$ that may contain correlation contributions besides the usual ideal part [9].

[9] For a review, see J.P. Hansen and E. Smargiassi, in "Monte Carlo and Molecular Dynamics of Condensed Matter Systems", edited by K. Binder and G. Ciccotti (SIF, Bologna, 1996), or R. Evans, in "Fundamentals of Inhomogeneous Fluids", edited by D. Henderson (Marcel Dekker, New York, 1992).

[10] M. Eigen and E. Wicke, J. Phys. Chem. 58, 702 (1954); V. Kralj-Iglič and A. Iglič, J. Phys. II France 6, 477 (1996); I. Borukhov, D. Andelman and H. Orland, Phys. Rev. Lett. 79, 435 (1997).

[11] L. Lue, N. Zoeller and D. Blankschtein, Langmuir 15, 3726 (1999).

[12] M.C. Barbosa, M. Deserno and C. Holm, cond-mat/9910364.

[13] L.D. Landau and E.M. Lifschitz, Electrodynamics of continuous media (Pergamon Press, Oxford, 1984). 
[14] B.I. Shklovskii, Phys. Rev. E 60, 5802 (1999); T. Das, D. Bratko, L.B. Bhuiyan and C.W. Outhwaite, J. Chem. Phys. 107, 9197 (1999); C.N. Patra and A. Yethiraj, J. Phys. Chem. B 103, 6080 (1999); P.S. Kuhn, Y. Levin and M.C. Barbosa, Physica A 274, 8 (1999).

[15] D. Goulding and J.P. Hansen, Europhys. Lett 46, 407 (1999).

[16] D. Goulding and J.P. Hansen, Mol. Phys. 95, 649 (1998).

[17] M.J. Stevens and M.O. Robbins, Europhys. Lett. 12, 81 (1990).

[18] A. Diehl, M.N. Tamashiro, M.C. Barbosa and Y. Levin, Physica A 274, 433 (1999).

[19] M.N. Tamashiro and P. Pincus, Phys. Rev. E 60, 6549 (1999). 